\title{
A Concise Review on Incidence and Risk Factors for Antituberculous Treatment-induced Hepatotoxicity
}

\author{
Kirthiga Lakshmi ${ }^{1}$, Vimal Raj $^{2}$
}

\begin{abstract}
Antituberculosis (anti-TB) drugs possess a variety of adverse effects, of which hepatotoxicity is the most common adverse effect as most of the anti-TB drugs are metabolized through the liver. Globally, the incidence of antituberculosis drug-induced hepatotoxicity (ATDH) ranges from $2 \%$ to $28 \%$ based on the definition of drug-induced liver injury, population of the study, and regimen of the treatment used. As documented in different studies, alcohol intake; elderly age; chronic liver disease; infections with hepatitis B virus (HBV), HCV, and HIV; advancement of TB; Asian ethnicity; female gender; concomitant use of enzyme-inducers; and poor nutritional status, were the possible risk factors for ATDH. Hepatic transaminase elevation without clinical presentation is a common and benign episode following anti-TB treatment, but symptomatic hepatotoxicity can be fatal without any intervention. Hepatotoxicity caused by antituberculous treatment (ATT) due to the combined use of drugs and long therapy period has been a major concern for clinical treatment. The rate of the liver injury due to ATT in developed and developing countries has been reported to be $4 \%$ and $39 \%$, respectively. Hence, there is an urgent need to review the risk factors and predictors of hepatotoxicity identified in various studies so as to plan reliable clinical interventions for disease prevention or timely identification and to develop safer drug regimens.
\end{abstract}

Keywords: Antituberculosis drug-induced hepatotoxicity, antituberculous treatment, Hepatotoxicity, Tuberculosis.

Annals of SBV (2019): 10.5005/jp-journals-10085-8108

\section{INTRODUCTION}

In 1993, the World Health Organization (WHO) declared TB to be a global emergency and still it continues to remain as a significant threat across the world. India accounts for one fourth of the TB burden, i.e., 2.2 million of the 9.6 million cases globally. ${ }^{1}$ In 1997, Revised National Tuberculosis Control Program (RNTCP) launched directly observed treatment shortcourse (DOTS) strategy which was internationally recommended. ${ }^{2}$ Thrice weekly regimens with the standard four-drug (HRZE) intermittent regimen was used initially. To lower the emergence of the drug-resistance TB and for simplicity of treatment, intermittent regimen has been changed to daily regimen with fixed dose combination (FDC) of drugs based on the weight band, from $2016 .^{2}$

Treatment with multiple drugs is important in the treatment of TB and to prevent the selection of the drug-resistant mutant bacilli. The antituberculous treatment (ATT) drugs used in FDC formulations ensure that the adequacy of the dose remains within the therapeutic range. ${ }^{3}$ The entire standard anti-TB treatment regimen consists of two phases, namely, intensive and continuation phase. The initial intensive phase is aimed at destroying the rapidly multiplying and semidormant bacilli and usually contains three to five drugs. The intensive phase of the standard primary treatment regimen includes four drugs, namely isoniazid $(H)$, rifampicin $(R)$, pyrazinamide ( $Z)$, and ethambutol (E). A standard four-drug regimen decreases the risk of development of drug resistance and plays a pivotal role in reducing the rates of relapse and failure. The continuation phase is aimed at destroying the most residual bacilli and in reducing failure and relapse rates. The continuation phase of the primary standard treatment is provided with three drugs, i.e., HRE. ${ }^{4}$

Anti-TB drugs possess a variety of adverse effects, of which hepatotoxicity is one of the most common adverse effects. The common adverse reactions of isoniazid include cutaneous reactions,
1,2Department of Pulmonary Medicine, Mahatma Gandhi Medical
College and Research Institute, Puducherry, India

Corresponding Author: Vimal Raj, Department of Pulmonary Medicine, Mahatma Gandhi Medical College and Research Institute, Puducherry, India, Phone: +91 9786652492, e-mail: iniyaulagam@ gmail.com

How to cite this article: Lakshmi K, Raj V. A Concise Review on Incidence and Risk Factors for Antituberculous Treatment-induced Hepatotoxicity. Ann SBV 2019;8(2):38-41.

Source of support: SBV PG Research Grant

Conflict of interest: None

lethargy, peripheral neuropathy, and hepatitis. The common adverse events associated with rifampicin are gastrointestinal reactions (such as nausea, vomiting, and abdominal pain), hepatitis, and cutaneous reactions. Arthralgia and hepatitis are the common adverse drug reactions (ADRs) associated with pyrazinamide. The main adverse reaction of ethambutol is retrobulbar neuritis. ${ }^{4}$ The spectrum of antituberculosis drug-induced hepatotoxicity (ATDH) is diverse ranging from asymptomatic rise in liver enzymes to fulminant hepatic failure. Anti-TB treatment-induced hepatotoxicity is one of the leading causes of liver injury in India as well as in other countries. It accounts for $58 \%$ of all cases of drug-induced liver injury and $5-22 \%$ cases of drug-induced acute liver failure. ${ }^{5}$ When an ADR occurs as a result of anti-TB treatment, especially when severe, one or more drugs may have to be discontinued or treatment should be interrupted, resulting in multiple implications, particularly extended treatment duration and an increased risk of drug resistance, relapse, and treatment failure. So all patients should be closely monitored to immediately recognize major ADRs and activate proper therapeutic measures. To recognize the development of ATDH, clinicians should be aware of the various risk factors associated with the development 
of ATDH. Numerous risk factors have been reported globally based on the different study population and different diagnostic criteria for ATDH. This review article aims at presenting a concise review of the incidence, mechanism of hepatotoxicity caused by first-line antitubercular drugs in adults and the risk factors associated with the development of ATDH.

\section{Materials and Methods}

Studies were identified from an electronic search of PUBMED (20022018). Variants of key words such as "tuberculosis," "antitubercular agents," "hepatotoxicity," and "adverse effects" were used. References from articles identified were also searched for relevant publications. Only manuscripts in English were considered.

\section{Incidence of ATDH}

Anti-TB treatment-induced hepatotoxicity is of major concern to the clinicians treating TB patients with ATT. In spite of this fact, there is no universal standard definition for ATDH. Incidence of ATDH ranges from $3 \%$ to $47 \%$ based on the definition of drug induced liver injury (DILI) used, population of the study and regimen of the treatment used (Table 1). ${ }^{6,7}$

The variability of the clinical response to the TB treatment might be related to the pharmacokinetic differences mostly attributed to the variability of the activity of enzymes implicated in hepatic metabolism of isoniazid (INH); that is, $\mathrm{N}$-acetyltransferase 2 (NAT2) and cytochrome P450. "It has been argued that genes encoding NAT2 and CYP2E1 are polymorphic and single-nucleotide polymorphisms (SNPs) on their coding regions could alter their enzymatic activity, resulting in an inter individual variability of expression of the NAT2 and CYP2E1 enzymes". 8,9

\section{Mechanism of ATDH}

Rifampicin may itself be hepatotoxic, as observed in cases with positive rechallenge and in situations where it is used alone (as to relieve pruritus). Pyrazinamide exhibits a wide range of hepatotoxicity which includes idiosyncratic dose-dependent, sporting eosinophilia and granulomatous hepatitis. ${ }^{10}$ Rifampicin induces hepatic CYP450 and leads to an increase in metabolism of other compounds. Rifampicin increases the production of hydrazine by inducing the isoniazid hydrolase enzyme. Concomitant use of isoniazid and rifampicin is associated with increased risk of the development of ATDH. Thus, the cause of hepatotoxicity by rifampicin may be due to enzyme induction, but the exact mechanism is unknown and it is often unpredictable. The precise mechanism of pyrazinamide causing hepatotoxicity is also unclear. Ethambutol does not have biliary clearance and is not hepatotoxic. Plethora of studies focused on the adverse reactions of INH, ${ }^{11,12}$ a drug metabolized by hepatic NAT and cytochrome P450 2E1 (CYP2E1) to develop hepatotoxins ${ }^{13}$ and reactive oxygen species. ${ }^{14}$ Although the first-line anti-TB drugs are more secure than the second-line drugs, hepatotoxicity to INH, rifampicin (RMP), and ethambutol (EMB) are well-documented in various studies. ${ }^{15}$ In recent years, new analytical techniques, such as metabolomics, genomics, proteomics, and transcriptomics, have provided a new approach and platform for exploring the toxicity, mechanism of action, and safety evaluation of certain compounds. ${ }^{16-18}$

\section{Risk Factors for Developing ATDH}

Many risk factors have been proposed for the development of ATDH. According to the previous works done on ATDH risk factors,
Table 1: Comparison of incidence and risk factors for ATDH from literature

\begin{tabular}{|c|c|c|}
\hline Study & $\begin{array}{l}\text { Incidence of } \\
\text { ATDH (\%) }\end{array}$ & Risk factors \\
\hline Wang et al. ${ }^{12}$ & 35.48 & $\begin{array}{l}\text { Increased total serum bilirubin, } \\
\text { aspartate aminotransferase and } \\
\text { preexisting hepatitis }\end{array}$ \\
\hline Abbara et al. ${ }^{6}$ & 6.9 & $\begin{array}{l}\text { Low patient weight, HIV-1 } \\
\text { co-infection, higher baseline alkaline } \\
\text { phosphatase (ALP) and alcohol } \\
\text { intake. }\end{array}$ \\
\hline Mariai et al. ${ }^{7}$ & 47.4 & $\begin{array}{l}\text { No statistically significant risk factors } \\
\text { were identified }\end{array}$ \\
\hline Abera et al. ${ }^{19}$ & 8 & High alcohol intake \\
\hline Saha et al. ${ }^{10}$ & 9.48 & $\begin{array}{l}\text { No statistically significant risk factors } \\
\text { were identified }\end{array}$ \\
\hline Sun et $\mathrm{al}^{20}$ & 12.9 & $\begin{array}{l}\text { Advanced age more than } 60 \text { years, } \\
\text { hypoalbuminemia HBsAg/ } \\
\text { HbeAg-positive hepatitis B carrier } \\
\text { status and chronic alcoholism. }\end{array}$ \\
\hline
\end{tabular}

Lee et al. ${ }^{21} \quad 10.5 \quad$ No statistically significant risk factors

Isa et al. ${ }^{22} \quad 18.2 \quad$ No statistically significant risk factors

Soliman et al. ${ }^{23} \quad 3.2$

Naqvi et al. $^{24} \quad 34.14$

ere identified

Ethnicity and diabetes

Age $>35$ years, concomitant hepatotoxic drugs, male gender, extrapulmonary $\mathrm{TB}$, and malnutrition

Gaude et al. ${ }^{25} \quad 3.6$

Old age, hypoalbuminemia, alcoholism, and advanced nature of the disease

Singla et al. ${ }^{26}-$ (case $\quad$ Older age, poor nutritional control status including baseline study) hypoalbuminemia, extrapulmonary $\mathrm{TB}$, moderate to advanced disease on chest radiograph

Khalili et al. ${ }^{27} \quad 31.4 \quad$ Infection with HIV, hepatitis C, concomitant use of hepatotoxic drugs, increased baseline serum alanine aminotransferase, and aspartate aminotransferase level

Khadka et al. ${ }^{28} 35 \quad$ Poor nutrition, alcohol consumption, HIV co-infection

Makhlouf $\quad 15.0 \quad$ Preexisting liver disease, et al. ${ }^{29}$ malnourished patients, and those with hypoalbuminemia and extensive or open pulmonary TB

Marzuki et al. ${ }^{30} 9.7$ HIV co-infection and extrapulmonary TB

Anand et al. ${ }^{31} \quad 10.1$

Presence of HBV infection, underlying silent chronic liver disease and empirical treatment with ATT without adequate clinical evidence.

Shakya et al. ${ }^{32} 8 \quad$ Female gender, disease extent, and malnutrition

Teleman $\quad 5.30 \quad$ Age more than 60 years, abnormal et al. ${ }^{33}$ baseline transaminase/serum bilirubin levels and female gender

Sharma et al. ${ }^{10}$ - (case Older age, moderately/far advanced control disease, serum albumin $3.5 \mathrm{~g} / \mathrm{dL}$, study) absence of HLADQA $1 * 0102$ and presence of HLA-DQB $1{ }^{*} 0201$ 
prevalence varies based on the study population, treatment regimen, and the definition of ATDH used. Detection of risk factors aids in early detection of ATDH, prompt management of the ATDH, and also lessens the mortality and morbidity associated with ATDH.

\section{Demographic Factors}

Among the demographic data, the most widely accepted risk factors include older age group, female gender, and low nutritional status. Most of the studies identified elderly age as a risk factor $21,26,27,31,36$ for developing ATDH, but in certain studies even younger patients ${ }^{25}$ was found to be at risk of developing ATDH which may be explained by the possible association between younger age and high alcohol consumption. Older patients are possibly more susceptible to drug-induced hepatotoxicity due to decrease in drug clearance from the liver caused by liver size, changes in blood flow, and drug distribution and binding with age. Based on the gender, females were found to have more risk of developing ATDH ${ }^{31,32}$ but in one study male gender was identified as a risk factor for ATDH. ${ }^{25}$ Low nutritional status or low BMI was identified as an independent risk factor for developing ATDH.

\section{HIV Co-infection}

Many studies have attributed the presence of HIV co-infection as one of the important risk factors for developing ATDH. ${ }^{6,28,29,32}$ It is unknown whether it is due to the HIV-related immunosuppression or the occurrence of hepatitis due to other opportunistic infections or higher prevalence of poor nutritional status among HIV co-infected patients. Most of the antiretroviral drugs used are potentially hepatotoxic and the concomitant usage of hepatotoxic drugs for both the diseases and chronicity of the treatment explains the higher incidence of ATDH among HIV/TB co-infected patients.

\section{Preexisting Liver Diseases}

Infections with hepatitis $B$ and $C$ are very important causes for the development of chronic liver disease and associated with higher incidence of development of ATDH. ${ }^{10,21,28}$ Various studies identified as infection with hepatitis $B$ and $C$ as a risk factor for developing ATDH. Patients with preexisting liver disease were at higher risk of developing ATDH. ${ }^{12,29}$ Patients with higher transaminases and higher bilirubin levels at the baseline were also found to be at higher risk of developing ATDH. $6,12,28,31$

\section{Nature and Severity of the Disease}

Certain studies have reported TB patients with extrapulmonary disease have increased risk of developing ATDH, ${ }^{10,24,29}$ whereas few studies show reported higher risk of ATDH among patients with pulmonary TB. ${ }^{29}$ Severity of the disease is found to be directly proportional to the risk of developing ATDH, and it has been reported in few studies including the Indian studies. ${ }^{10,25,26,29,32}$ Also an Indian study reported the higher risk of ATDH among TB patients who were started with ATT empirically without any clinical evidence. $^{31}$

\section{Intoxication}

Intoxication with alcohol has been attributed as one of the potential risk factors for developing ATDH. Alcohol consumption as a risk factor for development of ATDH may be attributed because of enzyme induction.

\section{Others}

Concomitant use of hepatotoxic drugs along with ATT have been reported as a risk factor for ATDH. ${ }^{24}$ Other risk factors attributed to the development of ATDH include dosing schedules, presence of comorbidities such as diabetes, acetylation status, and predisposing genetic factors such as polymorphisms in cytochrome P450 2E1 and the glutathione $S$-transferase. In an Indian study done in All India Institute of Medical Sciences, New Delhi, the absence of HLADQA $1 * 0102$ and the presence of HLA-DQB1*0201 were identified as an independent risk factors for developing ATDH. ${ }^{10}$

\section{Conclusion}

Managing ATDH is of paramount importance among cases with any type of TB that includes pulmonary, extrapulmonary, miliary and disseminated TB. The exact mechanism by which ATDH occurs is still not very clear. Further multicentric prospective studies are required in this regard for clearly understanding the mechanism of ATDH. Anti-tuberculosis drugs-induced hepatotoxicity not only causes morbidity and mortality per se but also significantly contributes to the interruption of TB treatment. Various risk factors for development of ATDH have been reported globally and the prevalence of those risk factors varies among the study population, duration of treatment, and definition of ATDH used. To lessen the morbidity and mortality and to reduce the duration of interruption of treatment with ATT, early detection of ATDH is necessary. Identification of risk factors for development of ATDH aids in early diagnosis and treatment of ATDH for which the standard universally acceptable screening facilities for risk factors are required. So we need further risk factor assessment studies among large and different population groups. The chronicity of the treatment of TB is also an important factor to be considered. Improvement in enhancing the bactericidal effect and reducing the treatment duration should be emphasized. As there is recent switch of intermittent to daily dosing schedules in the treatment of TB, we are in need of large multicentric prospective studies on whether the change in the dosing regime has any effect on the adverse reaction profile including ATDH. This may enable the clinicians and policy makers to find a clear picture of ATDH among patients who are on ATT treatment to set a gold standard.

\section{References}

1. World Health Organization. Global tuberculosis report 2018. Geneva: World Health; 2019.

2. Revised National Tuberculosis Control Programme (RNTCP). Technical and operational guidelines for tuberculosis control in India. 2016.

3. Blomberg B, Spinaci S, Fourie B, Laing R. The rationale for recommending fixed-dose combination tablets for treatment of tuberculosis. Bull World Health Organ 2001;79(1):61-68.

4. Frieden T. Toman's tuberculosis. Case detection, Treatment and Monitoring 2004.

5. Devarbhavi H, Singh R, Patil M, Sheth K, Adarsh CK, Balaraju G. Outcome and determinants of mortality in 269 patients with combination anti-tuberculosis drug-induced liver injury. J Gastroenterol Hepatol 2013;28(1):161-167. DOI: 10.1111/j.14401746.2012.07279.x.

6. Abbara A, Chitty S, Roe JK, Ghani R, Collin SM, Ritchie A, et al. Druginduced liver injury from antituberculous treatment: a retrospective study from a large TB centre in the UK. BMC Infect Dis 2017;17(1):231. DOI: 10.1186/s12879-017-2330-z. 
7. Maria NI, Radji MA, Burhan E. The impact of antituberculosis druginduced hepatotoxicity to successful tuberculosis treatment in Indonesia. Asian J Pharm Clin Res 2017;10(11):194-198. DOI: 10.22159/ ajpcr.2017.v10i11.20386.

8. Fretland $A J$, Leff MA, Doll MA, Hein DW. Functional characterization of human $\mathrm{N}$-acetyltransferase 2 (NAT2) single nucleotide polymorphisms. Pharmacogenetics 2001;11(3):207-215. DOI: 10.1097/00008571-200104000-00004.

9. Zang Y,Doll MA, Zhao S, States JC, Hein DW. Functional characterization of single-nucleotide polymorphisms and haplotypes of human $\mathrm{N}$-acetyltransferase 2. Carcinogenesis 2007;28(8):1665-1671. DOI: 10.1093/carcin/bgm085.

10. Sharma SK, Balamurugan A, Saha PK, Pandey RM, Mehra NK. Evaluation of clinical and immunogenetic risk factors for the development of hepatotoxicity during antituberculosis treatment. Am J Respir Crit Care Med 2002;166(7):916-919. DOI: 10.1164/ rccm.2108091.

11. Li F, Lu J, Cheng J, Wang L, Matsubara T, Csanaky IL, et al. Human PXR modulates hepatotoxicity associated with rifampicin and isoniazid co-therapy. Nat Med 2013;19(4):418. DOI: 10.1038/nm.3104.

12. Wang $\mathrm{P}$, Pradhan $\mathrm{K}$, Zhong $\mathrm{XB}, \mathrm{Ma} \mathrm{X}$. Isoniazid metabolism and hepatotoxicity. Acta Pharm Sin B 2016;6(5):384-392. DOI: 10.1016/j. apsb.2016.07.014.

13. Huang YS, Chern HD, Su WJ, Wu JC, Chang SC, Chiang CH, et al. Cytochrome P450 2E1 genotype and the susceptibility to antituberculosis drug-induced hepatitis. Hepatology 2003;37(4):924930. DOI: 10.1053/jhep.2003.50144.

14. Yue J, Peng R, Chen J, Liu Y, Dong G. Effects of rifampin on CYP2E1dependent hepatotoxicity of isoniazid in rats. Pharmacol Res 2009;59(2):112-119. DOI: 10.1016/j.phrs.2008.10.006.

15. Sterling TR, Villarino ME, Borisov AS, Shang N, Gordin F, BlivenSizemore $\mathrm{E}$, et al. Three months of rifapentine and isoniazid for latent tuberculosis infection. N Engl J Med 2011;365(23):2155-2166. DOI: 10.1056/NEJMoa1104875.

16. Arwood MJ, Deng J, Drozda K, Pugach O, Nutescu EA, Schmidt S, et al. Anticoagulation endpoints with clinical implementation of warfarin pharmacogenetic dosing in a real-world setting: A proposal for a new pharmacogenetic dosing approach. Clin Pharmacol Ther 2017;101(5):675-683. DOI: 10.1002/cpt.558.

17. Clayton TA, Lindon JC, Cloarec O, Antti H, Charuel C, Hanton G, et al. Pharmaco-metabonomic phenotyping and personalized drug treatment. Nature 2006;440(7087):1073. DOI: 10.1038/nature04648.

18. Liu L, Huang C, Bian Y, Miao L. GC-MS based metabolomics of CSF and blood serum: Metabolic phenotype for a rat model of cefoperazoneinduced disulfiram-like reaction. Biochem Biophys Res Commun 2017;490(3):1066-1073. DOI: 10.1016/j.bbrc.2017.06.167.

19. Abera W, Cheneke W, Abebe G. Incidence of antituberculosisdrug-induced hepatotoxicity and associated risk factors among tuberculosis patients in Dawro Zone, South Ethiopia: A cohort study. Int J Mycobacteriol 2016;5(1):14-20.

20. Sun Q, Zhang Q, Gu J, Sun WW, Wang P, Bai C, et al. Prevalence, risk factors, management, and treatment outcomes of first-line antituberculous drug-induced liver injury: a prospective cohort study. Pharmacoepidemiol Drug Saf 2016;25(8):908-917.DOI: 10.1002/ pds.3988.

21. Lee CM, Lee SS, Lee JM, Cho HC, Kim WS, Kim HJ, et al. Early monitoring for detection of antituberculous drug-induced hepatotoxicity. Korean J Intern Med 2016;31(1):65. DOI: 10.3904/kjim.2016. 31.1.65.

22. Isa SE, Ebonyi AO, Shehu NY, Idoko P, Anejo-Okopi JA, Simji G, et al. Antituberculosis drugs and hepatotoxicity among hospitalized patients in Jos, Nigeria. Int J Mycobacteriol 2016;5(1):21-26. DOI: 10.1016/j.ijmyco.2015.10.001.

23. Soliman I, Ahmad RA, Rajah J, AI Zaabi MR, Hassan YR, Yaqoob M, et al. Anti-tuberculous drug-induced hepatotoxicity: A retrospective look onto Abu-Dhabi tertiary health care center Sheikh Khalifa Medical City (SKMC) tuberculosis patients over a 7 years' period. J Adv Med Med Res 2016. 1-9.

24. Naqvi IH, Mahmood K, Talib A, Mahmood A. Antituberculosis druginduced liver injury: an ignored fact, assessment of frequency, patterns, severity and risk factors. Open J Gastroenterol 2015;5(12):173. DOI: 10.4236/ojgas.2015.512027.

25. Gaude GS, Hattiholi J, Reyas AK. Risk factors for drug induced hepatitis under dots programme in general population. SAARC Journal of Tuberculosis, Lung Diseases and HIV/AIDS 2013;10(1):19-26. DOI: 10.3126/saarctb.v10i1.8673.

26. Singla R, Sharma SK, Mohan A, Makharia G, Sreenivas V, Jha B, et al. Evaluation of risk factors for antituberculosis treatment induced hepatotoxicity. Indian J Med Res 2010;132:81-86.

27. Khalili H, Dashti-Khavidaki S, Rasoolinejad M, Rezaie L, Etminani M. Anti-tuberculosis drugs related hepatotoxicity; incidence, risk factors, pattern of changes in liver enzymes and outcome. DARU Journal of Pharmaceutical Sciences 2015;17(3):163-167.

28. Khadka J, Malla P, Jha SS, Poudel SR. The study of drug induced hepatotoxicity in att patients attending in National Tuberculosis Center in Bhaktapur. SAARC Journal of Tuberculosis, Lung Diseases and HIV/AIDS 2009;6(2):17-21. DOI: 10.3126/saarctb.v6i2. 3053

29. Makhlouf HA, Helmy A, Fawzy E, El-Attar M, Rashed HAG. A prospective study of antituberculous drug-induced hepatotoxicity in an area endemic for liver diseases. Hepatol Int 2008;2(3):353-360. DOI: 10.1007/s12072-008-9085-y.

30. Marzuki OA, Fauzi ARM, Ayoub S, Kamarul Imran M. Prevalence and risk factors of anti-tuberculosis drug-induced hepatitis in Malaysia. Singapore Med J 2008;49(9):688-693.

31. Anand AC, Seth AK, Paul M, Puri P. Risk factors of hepatotoxicity during anti-tuberculosis treatment. Med J Armed Forces India 2006;62(1):45-49. DOI: 10.1016/S0377-1237(06)80155-3.

32. Shakya R, Rao BS, Shrestha B. Incidence of hepatotoxicity due to antitubercular medicines and assessment of risk factors. Ann Pharmacother 2004;38(6):1074-1079. DOI: 10.1345/aph.1D525.

33. Teleman MD, Chee CBE, Earnest A, Wang YT. Hepatotoxicity of tuberculosis chemotherapy under general programme conditions in Singapore. Int J Tuberc Lung Dis 2002;6(8):699-705. 